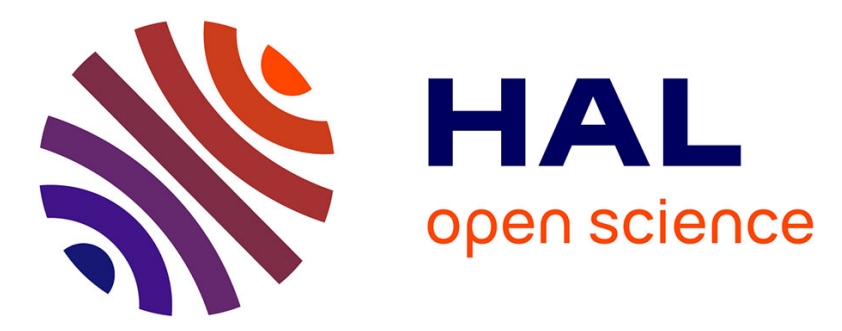

\title{
Blind Deconvolution of Medical Ultrasound Images Using a Parametric Model for the Point Spread Function
} Ningning Zhao, Qi Wei, Adrian Basarab, Denis Kouamé, Jean-Yves Tourneret

\section{To cite this version:}

Ningning Zhao, Qi Wei, Adrian Basarab, Denis Kouamé, Jean-Yves Tourneret. Blind Deconvolution of Medical Ultrasound Images Using a Parametric Model for the Point Spread Function. IEEE International Ultrasonics Symposium (IUS 2016), Sep 2016, Tours, France. pp. 1-4. hal-01517383

\section{HAL Id: hal-01517383 \\ https://hal.science/hal-01517383}

Submitted on 3 May 2017

HAL is a multi-disciplinary open access archive for the deposit and dissemination of scientific research documents, whether they are published or not. The documents may come from teaching and research institutions in France or abroad, or from public or private research centers.
L'archive ouverte pluridisciplinaire HAL, est destinée au dépôt et à la diffusion de documents scientifiques de niveau recherche, publiés ou non, émanant des établissements d'enseignement et de recherche français ou étrangers, des laboratoires publics ou privés. 


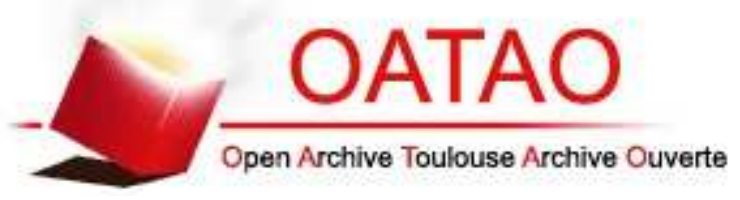

\section{Open Archive TOULOUSE Archive Ouverte (OATAO)}

OATAO is an open access repository that collects the work of Toulouse researchers and makes it freely available over the web where possible.

This is an author-deposited version published in : http://oatao.univ-toulouse.fr/ Eprints ID : 17011

The contribution was presented at IUS 2016 :

http://sites.ieee.org/ius-2016/

To cite this version : Zhao, Ningning and Wei, Qi and Basarab, Adrian and Kouamé, Denis and Tourneret, Jean-Yves Blind Deconvolution of Medical Ultrasound Images Using a Parametric Model for the Point Spread Function. (2016) In: IEEE International Ultrasonics Symposium (IUS 2016), 18 September 2016 - 21 September 2016 (Tours, France).

Any correspondence concerning this service should be sent to the repository administrator: staff-oatao@listes-diff.inp-toulouse.fr 


\title{
BLIND DECONVOLUTION OF MEDICAL ULTRASOUND IMAGES USING A PARAMETRIC MODEL FOR THE POINT SPREAD FUNCTION
}

\author{
Ningning Zhao ${ }^{1,2}$, Qi Wei ${ }^{3}$, Adrian Basarab ${ }^{2}$, Denis Kouamé ${ }^{2}$, Jean-Yves Tourneret ${ }^{1}$ \\ ${ }^{1}$ University of Toulouse, IRIT/INP-ENSEEIHT, 31071 Toulouse Cedex 7, France \\ ${ }^{2}$ University of Toulouse, IRIT, CNRS UMR 5505, Université Paul Sabatier, Toulouse, France \\ ${ }^{3}$ University of Cambridge, Department of engineering, UK \\ \{nzhao, jean-yves.tourneret\}@enseeiht.fr, \{qw245\}@cam.ac.uk, \{adrian.basarab, denis.kouame\}@irit.fr
}

\begin{abstract}
This paper addresses the problem of blind deconvolution of medical ultrasound (US) images. Specifically, a parametric model for the point spread function (PSF) established experimentally is used, i.e., the US PSF can be modeled by a Gaussian function modulated by a sinusoidal function. Given this parametric model, the estimation of the PSF in a blind deconvolution problem can be reduced to the estimation of its parameters. Moreover, due to the ill-posedness of blind deconvolution problem, an $\ell_{p}$-norm $(0<p \leqslant 2)$ regularization term (including the widely considered $\ell_{1}$-norm, $\ell_{2}$-norm regularization terms) for the ultrasound tissue reflectivity function (TRF) is employed, based on the assumption of generalized Gaussian distributed US images. An alternating optimization approach is proposed for the estimations of the US PSF and TRF. The behavior of the proposed algorithm is illustrated using simulated and in vivo US data.
\end{abstract}

Index Terms - Ultrasound imaging, blind deconvolution, block circulant matrix, optimization, variable metric forward backward splitting, proximal alternating linearized minimization.

\section{INTRODUCTION}

Medical ultrasound (US) imaging is widely used for clinical diagnosis such as cardiovascular medicine, urology and obstetrics. Compared to other medical imaging modalities, e.g., $\mathrm{X}$-ray computed tomography (CT) and magnetic resonance imaging (MRI), US imaging has many advantages, including its harmless, cost-effective, portable and noninvasive properties. However, US images suffer from a relatively low contrast, reduced spatial resolution at a given frequency and low signal-to-noise ratio (SNR). Even though advances in ultrasonic device-based solutions have improved the resolution of

Part of this work has been supported by the Chinese Scholarship Council and the thematic trimester on image processing of the CIMI Labex, Toulouse, France, under grant ANR-11-LABX-0040-CIMI within the program ANR11-IDEX-0002-02.
US images during the last decades, e.g., [1,2], post-processing techniques enhancing US image resolution are still appealing.

In this paper, we explore a blind deconvolution method aiming at improving the quality of US images. The linear model used for US image blind deconvolution can be defined using the following matrix-vector formulation

$$
\mathbf{y}=\mathbf{H x}+\mathbf{n}
$$

where $\mathbf{y}$ and $\mathbf{x}$ are vectors of $\mathbb{R}^{N \times 1}$ obtained after lexicographical order of the ultrasound radio-frequency (RF) image/observation and tissue reflectiviy function (TRF)/image to be estimated respectively, $\mathbf{n}$ is an additive white Gaussian noise (AWGN) and $\mathbf{H} \in \mathbb{R}^{N \times N}$ is the system impulse response/point spread function (PSF) assumed to be a circulant matrix $[3,4]$. In US imaging systems, the PSF is usually unknown. Existing methods to address this problem include either the estimation of the PSF in a pre-processing step [3,5] or the estimation of the PSF and the TRF simultaneously [6,7]. In this paper, we follow the second strategy to estimate the US TRF and PSF jointly. In particular, a parametric model for the PSF of the form of a modulated 2D Gaussian function is proposed. This parametric model allows us to reduce the estimation of the PSF during the blind deconvolution process to the estimation of a few parameters of the PSF model. In addition, a generalized Gaussian distribution is proposed for the US TRF [3]. It includes the widely used $\ell_{1}$-norm and $\ell_{2}$ norm regularizers in US image deconvolution literature, see e.g. $[8,9]$.

This paper is organized as follows. Section 2 introduces the proposed parametric model for the PSF and the formulated problem for US image blind deconvolution. The proposed alternating method is presented in Section 3. Section 4 displays the simulation results and conclusions are reported in Section 5. 


\section{PROBLEM STATEMENT}

\subsection{PSF parametric model}

We propose the following parametric model for the PSF of an US imaging system

$$
\mathbf{h}_{\mathrm{p}}(i, j) \equiv \mathbf{e}(i, j) \cos \left[\omega_{0} \mathbf{t}_{\mathrm{a}}(i)+\phi\right]
$$

with

$$
\mathbf{e}(i, j)=\mathbf{t}_{\mathbf{a}}^{3}(i) \exp \left[-\alpha \mathbf{t}_{\mathbf{a}}^{2}(i)\right] \exp \left[-\beta \mathbf{t}_{\mathbf{l}}^{2}(j)\right]
$$

where the parametric model of PSF " $h_{\mathrm{p}}$ " and its envelope " $\mathrm{e}$ " belong to $\mathbb{R}^{q \times r}$, the integers $i \in\{1, \cdots, q\}, j \in\{1, \cdots, r\}$ denote the location of the PSF pixels, $\omega_{0}=2 \pi f_{0}$ is the angular central frequency of the transducer, $\phi$ is the phase of the system PSF, the variables $\alpha, \beta$ determine the envelope shape of the PSF, the vectors $\mathbf{t}_{\mathrm{a}}$ and $\mathbf{t}_{1}$ are the temporal axes along the axial and lateral directions, which related to the PSF band widths. Thus, the vectors $\mathbf{t}_{\mathrm{a}} \in \mathbb{R}^{q \times 1}$ and $\mathbf{t}_{1} \in \mathbb{R}^{1 \times r}$ determine the size of the PSF. Note that a similar model was considered in [6], where a Gaussian function modulated by a sinusoidal function has been shown to fit well the US PSFs.

With the a priori knowledge of the temporal axes or the size of the $\mathrm{PSF}^{1}$, there are three parameters $\phi, \alpha, \beta$ to be estimated to completely determine the US PSF. The assumptions on the unknown PSF parameters considered herein are detailed hereafter.

- $\alpha$ and $\beta$ : We denote $\boldsymbol{\theta}=\{\alpha, \beta\}$. In this paper, we mainly focus on the estimation of the envelope shape parameters $\boldsymbol{\theta}$. Moreover, since the estimation of the two envelope shape parameters is ill-posed, we propose to constrain them as follows

$$
\begin{aligned}
& \rho(\alpha)=\imath_{\mathcal{C}_{\alpha}} \\
& \varrho(\beta)=\imath_{\mathcal{C}_{\beta}}
\end{aligned}
$$

where $\rho(\alpha)$ and $\varrho(\beta)$ are two indicator functions on sets $\mathcal{C}_{\alpha}=\left\{\alpha \in\left[\alpha_{\min }, \alpha_{\max }\right]\right\}$ and $\mathcal{C}_{\beta}=\left\{\beta \in\left[\beta_{\min }, \beta_{\max }\right]\right\}$. The definition of an indicator function is given by

$$
\imath_{\mathcal{C}}(x)= \begin{cases}0 & x \in \mathcal{C} \\ +\infty & x \notin \mathcal{C}\end{cases}
$$

- $\phi$ : In this paper, we estimate the phase term previously using the cepstrum-based method that exploits the minimum phase assumption of US systems [10]. However, we emphasis that it is possible to pass by the estimation of $\phi$ by dealing with complex demodulated signals following [6].

\footnotetext{
${ }^{1}$ The values of $q$ and $r$ or the size of the PSF are commonly assumed to be known in advance in the problem of US image deconvolution. Moreover, since the size of the PSF is usually much smaller compared with the image size (i.e., $q \ll m, r \ll n$ ), zero padding of the PSF is necessary for the convolution computation. Without loss of generality, all the PSFs mentioned in this paper have been zero padded for the convolution computation.
}

\subsection{Problem formulation}

Taking into account the parametric model for the PSF, we formulate the US image blind deconvolution problem as following

$$
\begin{array}{cl}
\min _{\mathbf{x}, \boldsymbol{\theta}} & \Psi(\mathbf{x}, \mathbf{h})+\tau \varphi(\mathbf{x})+\rho(\alpha)+\varrho(\beta) \\
\text { subject to } & \mathbf{h}=\mathbf{h}_{\mathbf{p}} .
\end{array}
$$

where $\Psi(\mathbf{x}, \mathbf{h})$ is the data fidelity term, $\varphi(\mathbf{x})$ is the regularization term for the TRF and $\tau$ is the corresponding regularization parameter which weights the importance between the data fidelity term and the regularization term. Under the assumption of additive white Gaussian noise (AWGN), we have

$$
\Psi(\mathbf{x}, \mathbf{h})=\frac{1}{2}\|\mathbf{y}-\mathbf{H} \mathbf{x}\|^{2} .
$$

Given a generalized Gaussian distribution as the prior information of US TRF [11, 12], we have

$$
\varphi(\mathbf{x})=\|\mathbf{x}\|_{p}^{p}
$$

\section{PROPOSED METHOD}

In order to solve the problem (7), we propose an alternating minimization approach following the block-coordinate descent (BCD) framework [13]. Algorithm 1 outlines the proposed approach.

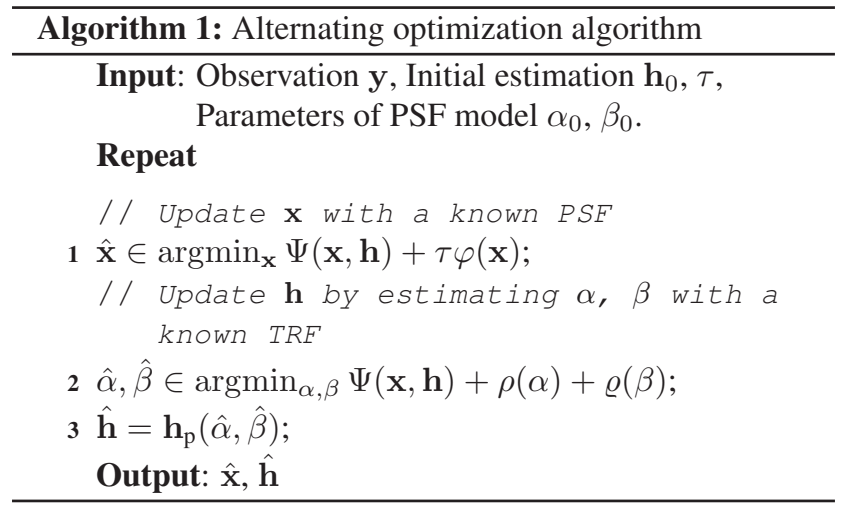

We note that the steps $\sharp 1$ and $\sharp 2$ can be solved using a proximal algorithm. More related details about this algorithm can be found in $[13,14]$.

\section{SIMULATION RESULTS}

In order to study the performance of the proposed algorithm, experiments have been conducted on simulated and in vivo ultrasound images. Moreover, a comparison with a non-blind deconvolution algorithm, where the PSF is estimated in a preprocessing step using the cepstrum-based algorithm $[10,15]$ 
has been conducted. For simulated US images, the performance of the algorithms is evaluated through the normalized root mean square error (NRMSE). The ground truth for the ultrasound TRF and PSF are not available for real US images. Therefore, the visually inspection has been used to evaluate the performance of TRF estimation for real images.

\subsection{Simulated US images}

Simulated ultrasound TRF of size $275 \times 75$ has been generated according to generalized Gaussian distribution, as shown in Fig. 1(b). The pixels in the different regions are distributed according to GGDs with different parameters: $p=1.2$ for the bright region, $p=1.8$ for the darker region surrounded by the bright zone and $p=0.6$ for the background. More details about this way of TRF generation can be found in [3]. The observed image shown in Fig. 1(a) has been blurred by a simulated PSF (displayed in Fig. 1(d)) that was generated following the model (2) and contaminated by an additive white Gaussian noise with blurred signal-to-noise ratio (BSNR) equal to $30 \mathrm{~dB}$. The parameters of the model (2) are fixed at $\alpha=4.8, \beta=10$ and $\phi=3.2$. Figs. 1(f), 1(h) display the estimated PSFs using the cepstrum-based method and the proposed method. Figs. 1(c), 1(e) and 1(g) show the restored ultrasound TRFs using the true PSF, the estimated PSFs obtained with the cepstrum-based method [10] and the proposed method respectively. The prior used for the TRF is an $\ell_{p}$-norm regularization $(p=1)$ for all experiments related to simulated images. The TRFs estimated using the true PSF and the proposed method are visually very similar. The PSF obtained with the proposed method is also closer to the true PSF than the one obtained by the cepstrum-based method. The quantitative results displayed in Table 1 confirm the visual impression in terms of NRMSE. Thus, for the simulated US images, the proposed blind deconvolution algorithm provides better performance than the non-blind deconvolution algorithm using a PSF estimated with cepstrum-based method both visually and quantitatively.

Table 1. Blind deconvolution performance of simulated US images.

\begin{tabular}{c|c|c|c}
\hline & & \multicolumn{2}{|c}{ NRMSE } \\
\hline Method & Prior & $\mathbf{x}$ & $\mathbf{h}$ \\
\hline Non blind with (d) & $\ell_{1}$ & 0.97 & 0 \\
Non blind with (f) & $\ell_{1}$ & 1.44 & 1.70 \\
Proposed & $\ell_{1}$ & $\mathbf{1 . 0 9}$ & $\mathbf{0 . 0 4}$ \\
\hline
\end{tabular}

\subsection{Experimental images}

The proposed blind deconvolution algorithm has also been tested on real US images. In this case, an $\ell_{p}$-norm with $p=$ 1.5 has been employed to regularize the TRF estimation. In this experiment, an ultrasound image representing a mouse

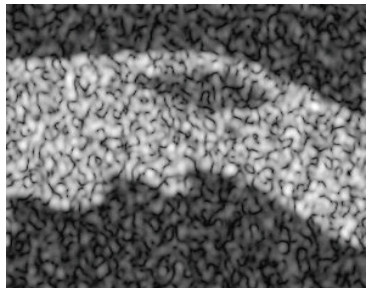

(a) Observation

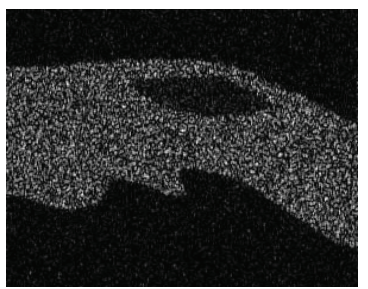

(c) Non blind with (d)

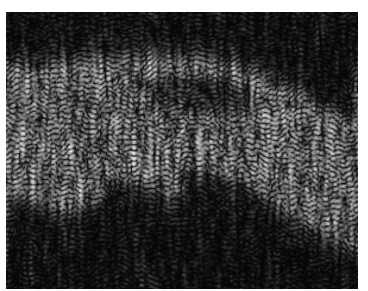

(e) Non blind with (f)

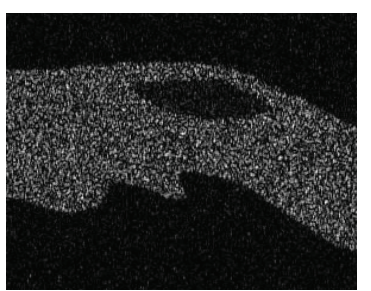

(g) Proposed

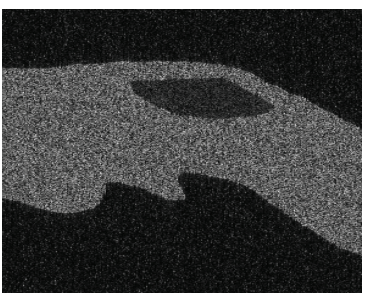

(b) Truth TRF

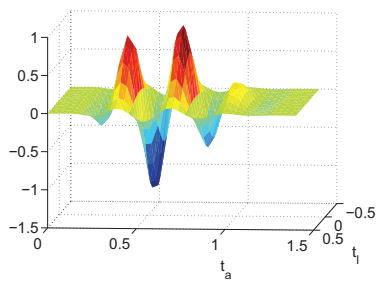

(d) Truth PSF

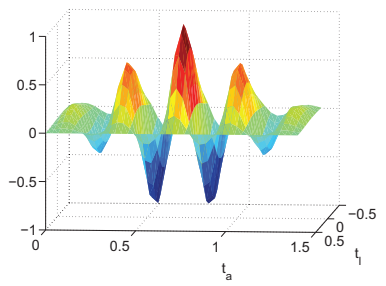

(f) Cepstrum

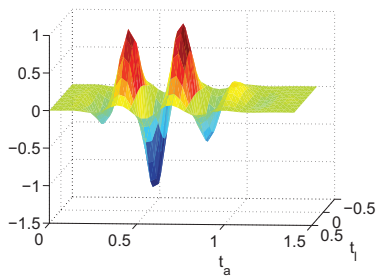

(h) Proposed
Fig. 1. Simulated US images.

kidney has been acquired with a $25 \mathrm{MHz}$ central frequency US probe, as shown in Fig. 2(a). The restored ultrasound TRFs shown in Figs. 2(b), 2(c) are obtained with the nonblind (cepstrum-based method) and the proposed algorithm. The estimated TRFs in Figs. 2(b), 2(c) have better defined boundaries than the observed image in Fig. 2(a). Moreover, the estimated TRF with the proposed algorithm provides competitive performance in terms of visual impression compared with the non-blind deconvolution algorithm.

\section{CONCLUSION}

This paper studied a new blind deconvolution algorithm for ultrasound images based on a parametric model of the PSF. By exploring an alternating optimization algorithm, we were able to calculate the maximum a posteriori estimations of 


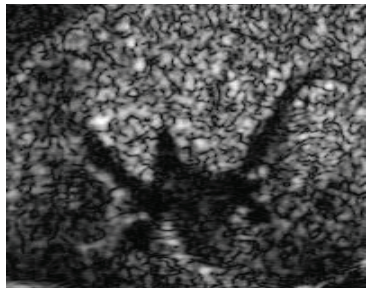

(a) Observation

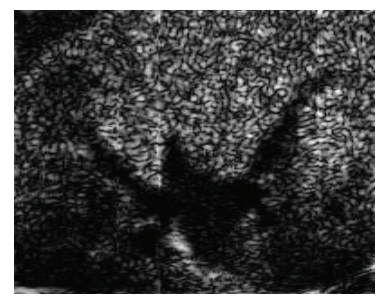

(b) Cepstrum $\left(\ell_{1.5}\right)$

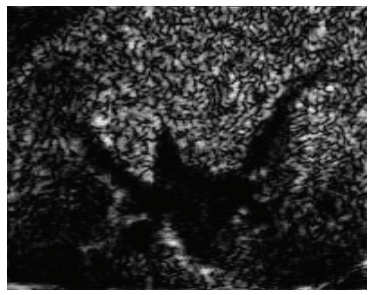

(c) Proposed $\left(\ell_{1.5}\right)$
Fig. 2. Real US images.

the ultrasound tissue reflectivity function and the system PSF simultaneously. Due to the parametric model of the PSF, instead of estimating all the PSF pixels, only a few parameters need to be estimated. This reduces the computational load and estimation complexity. Future work will be devoted to extend the proposed approach to complex envelope data/demodulated signals and to conduct more experiments on real ultrasound images.

\section{REFERENCES}

[1] M. A. Ellis, F. Viola, and W. F. Walker, "Superresolution image reconstruction using diffuse source models," Ultrasound in Med. and Bio., vol. 36, no. 6, pp. 967-977, 2010.

[2] M. Tanter and M. Fink, "Ultrafast imaging in biomedical ultrasound," IEEE Trans. Ultrason. Ferroelectr. Freq. Control, vol. 61, no. 1, pp. 102-119, 2014.

[3] N. Zhao, A. Basarab, D. Kouamé, and J.-Y. Tourneret, "Joint segmentation and deconvolution of ultrasound images using a hierarchical Bayesian model based on generalized Gaussian priors," IEEE Trans. Image Process., vol. 25, no. 8, pp. 3736 - 3750, 2016.

[4] N. Zhao, Q. Wei, A. Basarab, N. Dobigeon, D. Kouame, and J.-Y. Tourneret, "Fast single image super-resolution using a new analytical solution for $\ell_{2}-\ell_{2}$ problems," IEEE Trans. Image Process., vol. 25, no. 8, pp. 36833697, 2016.

[5] O. Michailovich and D. Adam, "A novel approach to the 2-D blind deconvolution problem in medical ultra- sound," IEEE Trans. Med. Imag., vol. 24, pp. 86-104, 2005 .

[6] C. Yu, C. Zhang, and L. Xie, "An envelope signal based deconvolution algorithm for ultrasound imaging," Signal Processing, vol. 92, no. 3, pp. 793 - 800, 2012.

[7] — , "A blind deconvolution approach to ultrasound imaging," IEEE Trans. Ultrason. Ferroelectr. Freq. Control, vol. 59, no. 2, pp. 271-280, 2012.

[8] R. Jirik and T. Taxt, "Two dimensional blind Bayesian deconvolution of medical ultrasound images," IEEE Trans. Ultrason. Ferroelectr. Freq. Control, vol. 55, no. 10, pp. 2140-2153, 2008.

[9] O. Michailovich and A. Tannenbaum, "Blind deconvolution of medical ultrasound images: A parametric inverse filtering approach," IEEE Trans. Image Process., vol. 16, no. 12, pp. 3005-3019, 2007.

[10] J. A. Jensen and S. Leeman, "Nonparametric estimation of ultrasound pulses," IEEE Trans. Biomed. Eng., vol. 41, no. 10, pp. 929-936, Oct. 1994.

[11] M. Alessandrini, A. Palladini, L. D. Marchi, and N. Speciale, "Expectation maximization for joint deconvolution and statistics estimation," Acoustical Imaging, vol. 30, no. 11, pp. 335-343, 2011.

[12] N. Zhao, Q. Wei, A. Basarab, D. Kouame, and J.Y. Tourneret, "Single image super-resolution of medical ultrasound images using a fast algorithm," in Proc. IEEE International Symposium on Biomedical Imaging (ISBI), Prague, CZ, April 2016.

[13] J. Bolte, S. Sabach, and M. Teboulle, "Proximal alternating linearizad minimization for noncovex and nonsmooth problems," Math. Program., pp. 459-494, Aug. 2014.

[14] A. Repetti, M. Q. Pham, L. Duval, E. Chouzenoux, and J.-C. Pesquet, "Euclid in a taxicab: Sparse blind deconvolution with smoothed $\ell_{1} / \ell_{2}$ regularization," IEEE Signal Process. Lett., vol. 22, no. 5, pp. 539-543, 2015.

[15] O. Michailovich and D. Adam, "Robust estimation of ultrasound pulses using outlier-resistant de-noising," IEEE Trans. Med. Imag., vol. 22, no. 3, pp. 368-381, 3 2003. 